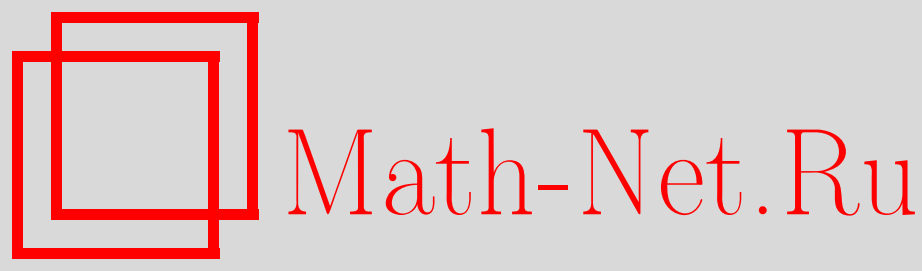

Д. А. Славнов, Проблема локальности в квантовой теории, ТМФ, 2008, том 155, номер 2, 327-343

DOI: https://doi.org/10.4213/tmf6215

Использование Общероссийского математического портала Math-Net.Ru подразумевает, что вы прочитали и согласны с пользовательским соглашением http://www . mathnet.ru/rus/agreement

Параметры загрузки :

IP : 3.93 .64 .190

26 апреля 2023 г., 16:20:49

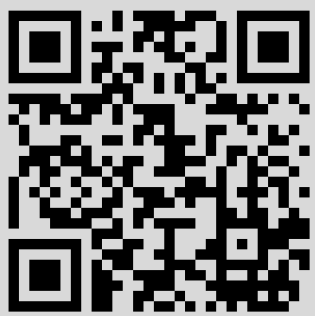




\title{
ПРОБЛЕМА ЛОКАЛЬНОСТИ В КВАНТОВОЙ ТЕОРИИ
}

\begin{abstract}
Обсуждается проблема локальности в релятивистской и нерелятивистской квантовой теории. Показано, что существует такая формулировка квантовой теории, которая, с одной стороны, сохраняет математический аппарат стандартной квантовой механики, с другой стороны, обеспечивает выполнение условия локальности для каждого индивидуального события, включая и процедуру измерения. В качестве примера рассмотрено рассеяние на двух щелях.
\end{abstract}

Ключевые слова: локальность измерения, алгебры локальных наблюдаемых, двухщелевой эксперимент, отсроченный выбор.

\section{1. ВВЕДЕНИЕ}

Со времен знаменитых дебатов Эйнштейна [1]-[3] и Бора [4], [5] среди специалистов ведутся споры о локальности в квантовой физике. Эйнштейн утверждал, что в квантовой механике нарушается принцип локальности. Поэтому она не последовательна или, по крайней мере, не полна. Основное возражение Бора сводилось к тому, что в квантовой теории физическую реальность и, соответственно, локальность нельзя трактовать так, как это делает Эйнштейн.

В значительной мере противоречия между Эйнштейном и Бором проистекали из-за различия во взглядах на основную задачу физики. Эйнштейн считал, что основная задача - понять и описать, как устроен материальный мир. По мнению Бора, основная задача - сформулировать правила, с помощью которых один специалист сможет сообщить другому, что надо делать, чтобы повторить эксперимент и получить тот же результат.

В годы, последовавшие за дебатами, Эйнштейну не удалось сколько-нибудь продвинуться по намеченному пути в квантовой теории. В противоположность этому сторонники Бора очень успешно решали поставленную им задачу.

В результате в научном сообществе прочно утвердилось мнение, что в споре прав был Бор. Точнее, подавляющее число специалистов, работающих в области квантовой физики, предпочитают не вникать в существо этого спора, считая его чисто философской проблемой, не заслуживающей внимания. Однако, поскольку они успешно используют правила, выработанные сторонниками Бора, их автоматически

* Московский государственный университет им. М. В. Ломоносова, Москва, Россия. E-mail: slavnov@goa.bog.msu.ru 
причисляют к лагерю его последователей. С другой стороны, большинство из них считают, что решают задачу описания действительности. Такая двойственная позиция не могла не отразиться на квантовой физике. В вопросе локальности запутанной оказалась связь между релятивистской квантовой теорией поля и нерелятивистской квантовой механикой.

В квантовой теории поля свойство локальности занимает центральное место. В подходе Вайтмана [6] так называемая эйнштейновская локальность является одним из основных постулатов. В подходе Боголюбова [7] основным конструктивным элементом служит условие причинности. Это условие теснейшим образом связано с локальностью, так как основывается на том, что любое возмущение в одной области пространства Минковского никак не влияет на физические процессы в другой пространственноподобной области. Наконец, алгебраический подход, связанный в первую очередь с именами Хаага [8] и Араки [9], целиком основывается на алгебрах локальных наблюдаемых, т.е. наблюдаемых, которые могут быть измерены в ограниченной области пространства Минковского.

В нерелятивистской квантовой механике свойство локальности взаимодействия квантовых объектов не отрицается. Однако ситуация коренным образом меняется при обсуждении вопроса о взаимодействии квантовых объектов с классическими измерительными приборами. Основным конструктивным элементом описания такого взаимодействия служит проекционный принцип [10]. Действительно, в большинстве случаев проекционный принцип оказывается очень полезным инструментом при описании в рамках стандартного математического аппарата квантовой механики воздействия измерительного прибора на квантовый объект. Однако сколько-нибудь внятного физического механизма реализации этого принципа и его согласования с условием локальности не предложено.

Вместо этого предлагаются туманные рассуждения о том, что человеческий мозг имеет опыт описания только классических объектов и поэтому не может дать наглядную картину взаимодействия квантового объекта с классическим прибором. Хотя человеческий мозг дает более или менее наглядную картину взаимодействия квантовых объектов между собой, но почему-то пасует в случае взаимодействия квантовых объектов с классическим прибором.

В последние годы проблема локальности становится все более и более актуальной. Это связано с тем, что она все больше переходит из области общетеоретических рассуждений и мысленных экспериментов в область реальных экспериментов. Более того, уже делаются первые попытки создания прототипов инженерных сооружений в области так называемой квантовой телекоммуникации. В этой области проблема квантовой локальности играет ключевую роль.

Здесь уместно сказать, что в большинстве случаев результаты современных экспериментов интерпретируются как свидетельство того, что в области квантовой физики "локальная физическая реальность" не существует. В таком случае локальность квантовой теории приобретает какой-то метафизический статус, оторванный от материальной действительности.

В настоящей работе будет предпринята попытка показать, что существует такая формулировка квантовой теории, которая, с одной стороны, сохраняет матема- 
тический аппарат стандартной квантовой механики, с другой стороны, допускает наглядную интерпретацию свойства локальности.

\section{2. ОСНОВНЫЕ ПОНЯТИЯ И ПОСТУЛАТЫ}

Основные понятия предлагаемого подхода к квантовой механике изложены в статье [11]. Более развернутое описание дано в [12]. Здесь мы ограничимся кратким обзором этих понятий. В [11] выбран индуктивный метод построения теории. Сначала рассматривается физическое явление, выделяются его основные характеристики, а затем дается их математическое описание.

В данной статье выбран дедуктивный метод, т.е. с самого начала формулируются математические постулаты. Интересующиеся их феноменологическим обоснованием могут обратиться к статьям [11] и [12]. Все построение ведется в рамках алгебраического подхода. Поэтому мы не предполагаем, что состояние физической системы описывается вектором гильбертова пространства (или матрицей плотности), а наблюдаемые - операторами в этом пространстве.

В качестве исходных примем следующие постулаты.

ПостулАт 1. Наблюдаемые физической системы описываются эрмитовыми элементами некоторой $C^{*}$-алгебры $\mathfrak{A}$.

Элементы алгебры $\mathfrak{A}$ будем называть динамическими величинами. Множество наблюдаемых обозначим $\mathfrak{A}_{+}$. Через $\mathfrak{Q}_{\xi}$ обозначим максимальные коммутативные подалгебры алгебры $\mathfrak{A}$, принадлежащие $\mathfrak{A}_{+}$. Индекс $\xi \in \Xi$ отличает одну такую подалгебру от другой.

ПостулАт 2. Чтобы наблюдаемые $\widehat{A}$ и $\widehat{B}$ были совместимы (одновременно измеримы), необходимо и достаточно, чтобы они принадлежали какой-нибудь одной подалгебре $\mathfrak{Q}_{\xi}$.

Обозначим через $\varphi_{\xi}(\cdot)$ характер подалгебры $\mathfrak{Q}_{\xi}$. То есть $\widehat{A} \stackrel{\varphi_{\xi}}{\longrightarrow} \varphi_{\xi}(\widehat{A})$ - гомоморфное отображение алгебры $\mathfrak{Q}_{\xi}\left(\widehat{A} \in \mathfrak{Q}_{\xi}\right)$ в алгебру действительных чисел.

Назовем элементарным состоянием физической системы совокупность $\varphi=\left[\varphi_{\xi}\right]$ $(\xi \in \Xi)$ функционалов $\varphi_{\xi}(\cdot)$, каждый из которых является некоторым характером соответствующей подалгебры $\mathfrak{Q}_{\xi}$.

ПостулАт 3. Результат каждого индивидуального эксперимента по измерению наблюдаемых физической системы определяется элементарным состоянием этой системы.

Одна и та же наблюдаемая может одновременно принадлежать нескольким подалгебрам $\mathfrak{Q}_{\xi}$. Будем говорить, что элементарное состояние $\varphi$ стабильно на наблюдаемой $\widehat{A}$, если для всех подалгебр $\mathfrak{Q}_{\xi}, \mathfrak{Q}_{\xi^{\prime}}$, содержащих $\widehat{A}$, справедливо равенство

$$
\varphi_{\xi}(\widehat{A})=\varphi_{\xi^{\prime}}(\widehat{A}), \quad \text { если } \quad \widehat{A} \in \mathfrak{Q}_{\xi} \cap \mathfrak{Q}_{\xi^{\prime}}, \quad \varphi_{\xi}(\cdot) \in \varphi, \quad \varphi_{\xi^{\prime}}(\cdot) \in \varphi .
$$

В общем случае условие (1) не выполняется. Это значит, что помимо элементарного состояния $\varphi$ результат измерения может зависеть от индекса $\xi \in \Xi$. Будем говорить, что прибор, предназначенный для измерения наблюдаемой $\widehat{A} \in \mathfrak{Q}_{\xi}$, 
принадлежит к $\xi$-типу, если, использовав его в качестве результата измерения, мы получаем $A_{\xi}=\varphi_{\xi}(\widehat{A})$. Таким образом, каждой подалгебре $\mathfrak{Q}_{\xi}$ ставится в соответствие определенный тип измерительных приборов. Именно с помощью приборов $\xi$-типа можно осуществить совместимые измерения наблюдаемых, принадлежащих подалгебре $\mathfrak{Q}_{\xi}$.

Измерение наблюдаемой $\widehat{A}$ назовем воспроизводимым, если при повторном измерении той же наблюдаемой (не обязательно прибором того же типа) получается результат, совпадающий с первоначальным. Очевидно, что в результате воспроизводимого измерения физическая система должна перейти в элементарное состояние, стабильное на наблюдаемой $\widehat{A}$.

Так как совместимые измерения можно провести только для совместимых наблюдаемых, то экспериментально мы не можем однозначно зафиксировать элементарное состояние $\varphi$. Максимум того, что мы можем сделать, - это определить сужение элементарного состояния на некоторую подалгебру $\mathfrak{Q}_{\xi}$, т.е. мы можем зафиксировать функционал $\varphi_{\xi}(\cdot)$. Элементарные состояния $\varphi$ назовем $\varphi_{\xi}$-эквивалентными, если они имеют одно и то же сужение $\varphi_{\xi}(\cdot)$ на подалгебру $\mathfrak{Q}_{\xi}$.

Чистым квантовым состоянием $\Psi_{\varphi_{\xi}}$ назовем класс $\{\varphi\}_{\varphi_{\xi}} \varphi_{\xi}$-эквивалентных элементарных состояний, стабильных на подалгебре $\mathfrak{Q}_{\xi}$. Таким образом, в эксперименте можно определить лишь принадлежность исследуемой системы к некоторому квантовому состоянию.

ЗАмЕЧАниЕ. Приведенное определение квантового состояния пригодно только для физических систем, в которых нет тождественных частиц. При наличии таких частиц в определении квантового состояния эквивалентность следует заменить на слабую эквивалентность (см. работу [12]).

Чистым квантовым ансамблем $\Psi_{\varphi_{\xi}}$ назовем множество физических систем, элементарные состояния которых образуют класс эквивалентности $\{\varphi\}_{\varphi_{\xi}}$.

ПостулАт 4. Квантовый ансамбль допускает введение структуры вероятностного пространства.

Напомним, что вероятностное пространство является основным понятием классической теории вероятностей (см., например, [13], [14]). Вероятностное пространство - это триада $(\Omega, \mathcal{F}, P)$. Первый член триады $\Omega$ - множество элементарных событий. У нас в качестве элементарных событий будут выступать элементарные состояния $\varphi$. Второй член триады $\mathcal{F}$ - булева $\sigma$-алгебра множества $\Omega$. Элементами алгебры $\mathcal{F}$ являются подмножества множества $\Omega$. Эти подмножества называются (вероятностными) событиями. Среди них должны обязательно присутствовать само множество $\Omega$ и пустое множество $\varnothing$. В качестве алгебраических в $\mathcal{F}$ рассматриваются такие операции: объединение подмножеств, их пересечение и дополнение до множества $\Omega$. Алгебра $\mathcal{F}$ должна быть замкнута (инвариантна) относительно дополнения и счетного числа объединений и пересечений. Третий член триады вероятностная мера $P$. Это отображение множества $\mathcal{F}$ в множество действительных чисел: каждому элементу $F$ алгебры $\mathcal{F}$ ставится в соответствие число $P(F)$. Отображение должно удовлетворять условиям $0 \leqslant P(F) \leqslant 1$ для каждого $F \in \mathcal{F}$, 
$P(\Omega)=1, P\left(\sum_{j} F_{j}\right)=\sum_{j} P\left(F_{j}\right)$ для каждого счетного объединения $\sum_{j} F_{j}$ непересекающихся подмножеств $F_{j} \in \mathcal{F}$.

Обратим внимание на то, что вероятностная мера определяется только для событий $F \in \mathcal{F}$. Для элементарных событий, в общем случае, вероятностная мера может не существовать. Это связано с тем, что прежде чем говорить о вероятности какого-то события, нужно, чтобы это событие могло быть (хотя бы в принципе) реализовано. Из-за конечности разрешающей способности измерительных приборов, вообще говоря, элементарное событие выделить нельзя. Можно убедиться (см., например, [12]), что предположение об обязательном существовании вероятностной меры элементарных событий в некоторых случаях ведет к противоречию.

В этом плане квантовая теория налагает еще более жесткие ограничения, чем классическая. Это связано с тем, что для выделения события мы должны иметь возможность произвести одновременное (или, по крайней мере, совместимое) измерение некоторой совокупности наблюдаемых. В квантовом случае совместимы только наблюдаемые, принадлежащие какой-то одной подалгебре $\mathfrak{Q}_{\xi}$. Отсюда следует, что каждой такой подалгебре $\mathfrak{Q}_{\xi}$ должна соответствовать своя $\sigma$-алгебра $\mathcal{F}_{\xi}$ и своя система вероятностных мер $P_{\xi}(F)$, где $F \in \mathcal{F}_{\xi}$.

С другой стороны, существуют события, которые могут быть выделены с помощью измерения наблюдаемых, принадлежащих разным подалгебрам $\mathfrak{Q}_{\xi}$. Примером такого события является событие $F_{A}$, заключающееся в том, что в эксперименте для некоторой наблюдаемой $\widehat{A}$ регистрируется значение, не большее $A$. Если $\widehat{A} \in \mathfrak{Q}_{\xi} \cap \mathfrak{Q}_{\xi^{\prime}}$, то это событие может быть зарегистрировано как приборами типа $\xi$, так и приборами типа $\xi^{\prime}$. Опыт показывает, что вероятность такого события не зависит от типа используемого прибора. Поэтому мы должны принять еще один постулат.

ПостулАт 5. Если $\widehat{A} \in \mathfrak{Q}_{\xi} \cap \mathfrak{Q}_{\xi^{\prime}}$, то для системы, находящейся в некотором квантовом состоянии $\Psi$, вероятность обнаружить событие $F_{A}$ не зависит от типа используемого прибора, т.е. $P\left(\varphi: \varphi_{\xi}(\widehat{A}) \leqslant A\right)=P\left(\varphi: \varphi_{\xi^{\prime}}(\widehat{A}) \leqslant A\right)$.

Математическим образом физической системы является алгебра ее динамических величин, и наоборот, физическим образом алгебры динамических величин является некоторая физическая система. Поэтому можно считать, что физическим образом подалгебры является соответствующая физическая подсистема. Конечно, эта подсистема не обязана быть изолированной от остальной части системы, т.е. она может быть открытой системой и не иметь собственной динамики. Однако в большинстве случаев выводы теории вероятностей никак не связаны с динамикой. В частности, подалгебру $\mathfrak{Q}_{\xi}$ можно рассматривать как алгебру наблюдаемых некоторой классической подсистемы изучаемой квантовой системы. Так как для нахождения среднего значения $\langle\widehat{A}\rangle$ наблюдаемой $\widehat{A} \in \mathfrak{Q}_{\xi}$ можно ограничиться измерениями, совместимыми с измерениями наблюдаемых из подалгебры $\mathfrak{Q}_{\xi}$, то для математического вычисления такого среднего достаточна классическая теория вероятностей. Это значит, что справедлива формула

$$
\langle\widehat{A}\rangle=\int_{\varphi \in \Psi} P_{\widehat{A}}(d \varphi) A(\varphi) \equiv \int_{\varphi \in \Psi} P_{\widehat{A}}(d \varphi) \varphi(\widehat{A}) .
$$


Здесь

$$
P_{\widehat{A}}(d \varphi)=P(\varphi: \varphi(\widehat{A}) \leqslant A+d A)-P(\varphi: \varphi(\widehat{A}) \leqslant A), \quad A_{\xi}(\varphi) \equiv \varphi_{\xi}(\widehat{A}) .
$$

В формулах $(2)$ и $(3)$, имея в виду постулат 5 , у функционалов $A_{\xi}(\varphi)$ и $\varphi_{\xi}(\widehat{A})$ мы можем опустить индекс $\xi$.

Формула (2) определяет среднее значение наблюдаемой $\widehat{A}$ по квантовому ансамблю. Экспериментально квантовое среднее $\Psi(\widehat{A})$ определяется как среднее арифметическое по результатам измерения наблюдаемой $\widehat{A}$. Связь между величинами $\langle\widehat{A}\rangle$ и $\Psi(\widehat{A})$ дается теоремой Хинчина (законом больших чисел, см., например, [14]), которая в терминах, используемых в данной статье, может быть сформулирована следующим образом.

Теорема. Пусть $A_{\xi_{j}}=\varphi_{\xi_{j}}(\widehat{A})$ - результат измерения наблюдаемой $\widehat{A}$ в эксперименте с номером $j$. Пусть $A_{\xi_{j}}$ - случайные взаимно независимые величины, имеющие одно и то же распределение вероятностей $P_{\widehat{A}}$ с конечным математическим ожиданием $\langle\widehat{A}\rangle$. Тогда при $n \rightarrow \infty$ величина $n^{-1}\left(A_{\xi_{1}}+\cdots+A_{\xi_{n}}\right)$ сходится по вероятности $\kappa\langle\widehat{A}\rangle$. Таким образом,

$$
\Psi(\widehat{A}) \equiv \lim _{n \rightarrow \infty} \frac{\varphi_{\xi_{1}}(\widehat{A})+\cdots+\varphi_{\xi_{n}}(\widehat{A})}{n}=\langle\widehat{A}\rangle .
$$

Эксперимент указывает, что справедливо следующее утверждение.

ПостулАт 6 . Величина $\Psi(\widehat{A})$ является линейным функционалом от наблюдаемых, т.е.

$$
\Psi(\widehat{A})+\Psi(\widehat{B})=\Psi(\widehat{A}+\widehat{B}) \quad \text { для всех } \widehat{A}, \widehat{B} \in \mathfrak{A}_{+} .
$$

На алгебру $\mathfrak{A}$ данный функционал однозначно расширяется в соответствии с формулой $\Psi(\widehat{A}+i \widehat{B})=\Psi(\widehat{A})+i \Psi(\widehat{B})$, где $\widehat{A}, \widehat{B} \in \mathfrak{A}_{+}$.

Каждая $C^{*}$-алгебра $\mathfrak{A}$ изометрически изоморфна подалгебре алгебры $\mathfrak{B}(\mathfrak{H})$ ограниченных линейных операторов в некотором гильбертовом пространстве $\mathfrak{H}$ (см., например, [15]), т.е.

$$
\widehat{A} \leftrightarrow \Pi(\widehat{A}), \quad \widehat{A} \in \mathfrak{A}, \quad \Pi(\widehat{A}) \in \mathfrak{B}(\mathfrak{H}) .
$$

Можно убедиться (см. [11], [12]), что среднее значение $\langle\widehat{A}\rangle$ наблюдаемой $\widehat{A}$ по квантовому ансамблю $\Psi$, определяемое формулой $(2)$, представимо в виде математического ожидания оператора П $(\widehat{A})$ :

$$
\langle\widehat{A}\rangle=\langle\Psi|\Pi(\widehat{A})| \Psi\rangle
$$

где $|\Psi\rangle \in \mathfrak{H}-$ соответствующий вектор гильбертова пространства.

\section{3. ЛОКАЛЬНОСТЬ НАБЛЮДАЕМЫХ И НЕЛОКАЛЬНОСТЬ СОСТОЯНИЙ}

Формулы (4) и (5) говорят, что, с одной стороны, для вычисления квантовых средних $\Psi(\widehat{A})$ можно использовать математический аппарат стандартной квантовой механики, с другой стороны, интерпретировать квантовое состояние как класс 
эквивалентности элементарных состояний. Класс эквивалентности - это абстрактное понятие, которое существует вне времени и пространства. Поэтому говорить о какой-то локализации квантового состояния бессмысленно.

Класс эквивалентности, соответствующий определенному квантовому состоянию, формируется по некоторым признакам, общим для всех элементов этого класса. Такими общими признаками являются одинаковые значения некоторых наблюдаемых, получаемые с помощью определенной измерительной процедуры. Ни время, ни координата не являются наблюдаемыми величинами. Это параметры пространства Минковского, в котором существуют физические объекты. Правда, в нерелятивистской квантовой механике очень часто используют наблюдаемую, которую называют "координатой".

Чтобы разобраться в такой путанице, рассмотрим более внимательно процедуру измерения этой "координаты". Для простоты рассуждений будем считать, что изучаемый физический объект точечный. Чтобы измерить "координату" физического объекта, мы используем измерительный прибор - линейку с нанесенными на нее делениями. С каждым промежутком между двумя соседними делениями ассоциируем наблюдаемую $\hat{p}_{i}$, где $i$ - номер соответствующего промежутка. Если изучаемый физический объект оказался внутри $i$-го промежутка, то наблюдаемой $\hat{p}_{i}$ приписываем значение 1 , если не оказался, то приписываем $\hat{p}_{i}$ значение 0. Определенная таким образом наблюдаемая $\hat{p}_{i}$ обладает свойствами проектора и является элементом алгебры динамических величин.

Допуская вольность речи, назовем индекс $i$ координатой и будем говорить, что изучаемый объект имеет координату $i$, если значение соответствующей наблюдаемой $\hat{p}_{i}$ оказалось равным 1 . K истинной координате, параметру в пространстве Минковского, "координата" $i$ имеет очень отдаленное отношение. Мы можем повторить наш опыт, перенеся его в другую область пространства Минковского. При этом мы должны перенести в эту область как изучаемый объект, так и линейку. Если в последнем случае изучаемый объект опять окажется в промежутке с номером $i$, то будем считать, что во втором эксперименте мы получили то же значение "координаты". Именно по этой “координате" (фактически по значению наблюдаемой $\hat{p}_{i}$ ) мы можем сформировать класс эквивалентности и сказать, что мы построили квантовое состояние, сосредоточенное в окрестности "координаты" $i$. К локализации в пространстве Минковского эта "координата" не будет иметь никакого отношения.

Чтобы более предметно обсудить проблему локальности, посмотрим, как с помощью идеологии элементарного состояния можно описать рассеяние частицы на двух щелях $a$ и $b$. В этом эксперименте отчетливо наблюдается интерференционная картина. Ясно, что эта картина определяется вероятностным распределением импульсов частиц после рассеяния. В рассматриваемом эксперименте существенную роль играют три события: событие $F_{a}$ - попадание частицы в область щели $a$, событие $F_{b}$ - попадание частицы в область щели $b$, событие $F_{k}$ - попадание импульса рассеянной частицы в фиксированный малый телесный угол вокруг направления $k$.

В этих терминах изучаемую проблему можно сформулировать как типичную задачу на вычисление условной вероятности. Надо вычислить вероятность события $F_{k}$ при условии реализации либо события $F_{a}$, либо $F_{b}$. На этот счет в классической тео- 
рии вероятностей существует стандартная формула. Однако в квантовом случае ее непосредственно применять нельзя, так как в ней фигурирует вероятность одновременной реализации событий $F_{k}$ и $F_{a}+F_{b}$. Но для такого события вероятностная мера не существует, так как события $F_{k}$ и $F_{a}+F_{b}$ несовместимы, поскольку несовместимы измерения координаты и импульса.

Однако можно предложить обходной путь для вычисления такой условной вероятности. Для этого достаточно рассмотреть первый этап рассеяния - попадание частицы либо в область щели $a$, либо в область щели $b$ - как приготовление некоторого квантового состояния. При использовании этого квантового состояния в качестве нового вероятностного пространства событие $F_{k}$ уже можно считать безусловным.

Событию $F_{a}$ сопоставим наблюдаемую $\hat{p}_{a}$, которая принимает значение $p_{a}=1$, если частица попала в область щели $a$, и значение $p_{a}=0$, если не попала. Событию $F_{b}$ сопоставим аналогичную наблюдаемую $\hat{p}_{b}$. В формировании интерференционной картины принимают участие только те частицы, элементарные состояния которых соответствуют значению наблюдаемой $\hat{p}_{a}+\hat{p}_{b}$, равному 1 . Такие элементарные состояния образуют класс эквивалентности, который мы обозначим $\Psi_{a+b}$. Поскольку в общем случае наблюдаемая $\hat{p}_{a}+\hat{p}_{b}$ является не единственным независимым генератором максимальной подалгебры совместимых наблюдаемых, то квантовое состояние, соответствующее классу эквивалентности $\Psi_{a+b}$, может быть смешанным. Однако и в этом случае функционал, описывающий среднее значение наблюдаемых в таком квантовом состоянии, будет положительным, линейным и нормированным на единицу. Этот функционал мы обозначим $\Psi_{a+b}(\cdot)$. Он будет обладать свойством

$$
\Psi_{a+b}(\hat{I})=1
$$

где $\hat{I}$ - единичный элемент алгебры $\mathfrak{A}$. Кроме того, для всех элементарных состояний, входящих в состав этого квантового состояния, $p_{a}+p_{b}=1$, поэтому в силу формул $(2)$ и (4) функционал $\Psi_{a+b}(\cdot)$ удовлетворяет условию

$$
\Psi_{a+b}\left(\hat{p}_{a}+\hat{p}_{b}\right)=1
$$

Так как функционал $\Psi_{a+b}(\cdot)$ положителен, для него справедливо неравенство Коши-Буняковского-Шварца

$$
\left|\Psi_{a+b}\left(\widehat{A}\left(\hat{I}-\hat{p}_{a}-\hat{p}_{b}\right)\right)\right|^{2} \leqslant \Psi_{a+b}\left(\widehat{A}^{*} \widehat{A}\right) \Psi_{a+b}\left(\hat{I}-\hat{p}_{a}-\hat{p}_{b}\right) .
$$

В силу равенств (6) и (7) правая часть неравенства (8) равна нулю. Поэтому

$$
\Psi_{a+b}(\widehat{A})=\Psi_{a+b}\left(\widehat{A}\left(\hat{p}_{a}+\hat{p}_{b}\right)\right) .
$$

Аналогично

$$
\Psi_{a+b}(\widehat{A})=\Psi_{a+b}\left(\left(\hat{p}_{a}+\hat{p}_{b}\right) \widehat{A}\right) .
$$

Делая в (10) замену $\widehat{A} \rightarrow \widehat{A}\left(\hat{p}_{a}+\hat{p}_{b}\right)$ и учитывая $(9)$, получаем

$$
\Psi_{a+b}(\widehat{A})=\Psi_{a+b}\left(\left(\hat{p}_{a}+\hat{p}_{b}\right) \widehat{A}\left(\hat{p}_{a}+\hat{p}_{b}\right)\right) .
$$


Событию $F_{k}$ сопоставим наблюдаемую $\widehat{K}$. Воспользовавшись формулой $(11)$, для среднего значения этой наблюдаемой получаем

$$
\langle\widehat{K}\rangle=\Psi_{a+b}(\widehat{K})=\Psi_{a+b}\left(\hat{p}_{a} \widehat{K} \hat{p}_{a}\right)+\Psi_{a+b}\left(\hat{p}_{b} \widehat{K} \hat{p}_{b}\right)+\Psi_{a+b}\left(\hat{p}_{a} \widehat{K} \hat{p}_{b}+\hat{p}_{b} \widehat{K} \hat{p}_{a}\right) .
$$

Первое и второе слагаемые в правой части (12) описывают рассеяние на щели $a$ и щели $b$ соответственно. Третье слагаемое описывает интерференцию. Поскольку $\hat{p}_{a} \hat{p}_{b}=\hat{p}_{b} \hat{p}_{a}=0$, то в случае, когда $\left[\hat{p}_{a}, \widehat{K}\right]=0$ или $\left[\hat{p}_{b}, \widehat{K}\right]=0$, интерференционный член исчезает.

Интерференционная картина целиком определяется структурой абстрактного класса эквивалентности $\Psi_{a+b}$. Поэтому мы могли бы организовать наш эксперимент следующим образом. Сделать много дубликатов одной и той же экспериментальной установки и разбросать их по всему Земному Шару. На каждой установке в произвольные моменты времени произвести по одному рассеянию. Потом собрать все экраны, на которых образовались пятна от попадания рассеянных частиц, и совместить все эти экраны. При достаточно большом количестве таких экранов мы должны получить картину, близкую к той, которая описывается формулой (12).

Отметим, что в отличие от рассмотрения того же эксперимента в стандартной квантовой механике, мы считали, что в каждом отдельном случае рассеивающаяся частица попала либо в область щели $a$, либо в область щели $b$, а не каким-то таинственным образом прошла одновременно через обе щели. Это значит, что мы считали, что в каждом отдельном случае частица хорошо локализована. Интерференционная картина возникает из-за того, что функционал $\Psi_{a+b}(\cdot)$ не представим в виде суммы функционалов $\Psi_{a}(\cdot)$ и $\Psi_{b}(\cdot)$, соответствующих рассеянию отдельно на щели $a$ и щели $b$. Физически это означает, что рассеяние на каждой из щелей зависит от того, имеется или не имеется другая щель, т.е. какая-то нелокальность здесь присутствует.

Посмотрим, как эту нелокальность можно объяснить в рамках локальной теории поля. Для большей наглядности обсуждение проведем на примере хорошо изученного как теоретически, так и экспериментально процесса рассеяния электрона на ядре. Так как электрон значительно легче ядра, то этот процесс хорошо аппроксимируется рассеянием электрона на классическом источнике. Именно этот процесс мы далее будем обсуждать в рамках теории возмущений стандартной квантовой электродинамики (см., например, [16]).

В первом порядке теории возмущений по заряду электрона этот процесс описывается диаграммой Фейнмана “а”, изображенной на рис. 1. На этом рисунке прямые линии соответствуют электрону, волнистые - фотону, кружок с крестиком - источнику классического электромагнитного поля. Расчет дифференциального сечения рассеяния при учете диаграммы "а" не вызывает никаких трудностей и приводит к известной формуле Резерфорда, подправленной с учетом наличия спина у электрона. Полученная формула хорошо описывает экспериментальную ситуацию. Однако сегодня и теория, и эксперимент давно перешагнули порог точности, который может обеспечить первый порядок теории возмущений.

Следующий порядок теории возмущений, который дает вклад в изучаемый процесс - это третий порядок. Здесь надо учесть вклады от диаграмм “б” и "в" на рис. 1. 


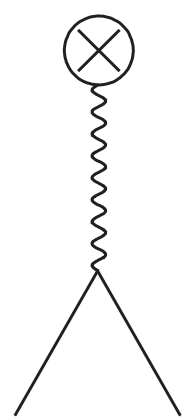

$a$

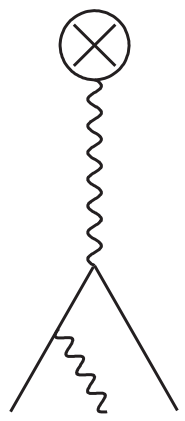

2

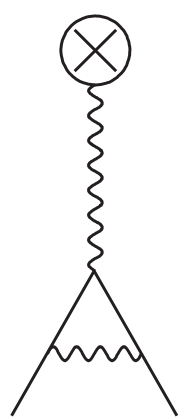

$\sigma$

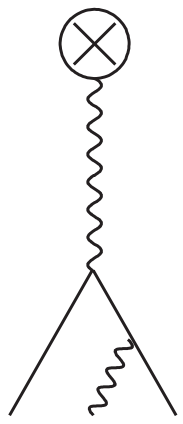

$\partial$

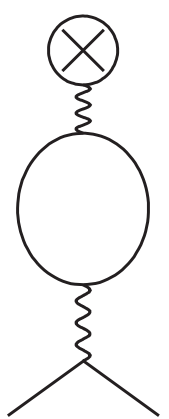

8

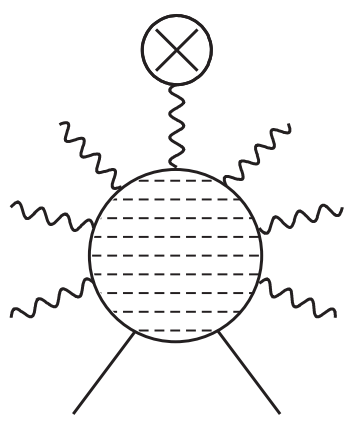

$e$

Рис. 1. Диаграммы, описывающие упругое рассеяние (а-в), и диаграммы, описывающие тормозное излучение (г-е).

Учет этих диаграмм приводит к значительным теоретическим трудностям. Во-первых, возникают так называемые ультрафиолетовые расходимости, обусловленные тем, что промежуточные (виртуальные) частицы могут переносить сколь угодно большие энергию и импульс. В квантовой теории поля разработан четкий алгоритм преодоления этой трудности (теория перенормировок). Далее обсуждать эту проблему мы не будем. Во-вторых, диаграмма "б" приводит к так называемым инфракрасным расходимостям, которые обусловлены тем, что среди частиц есть частицы с нулевой массой. В рассматриваемом примере - это фотоны. В квантовой теории поля есть алгоритм преодоления и этой трудности. На нем мы остановимся несколько подробнее.

Этот алгоритм основан на следующем экспериментальном факте. Процесс упругого рассеяния, который описывается диаграммами "а"-"в", экспериментально не отделим от процесса, изображенного на диаграммах "г"-“е” и называемого тормозным излучением. В этом процессе рассеяние электрона сопровождается испусканием одного (диаграммы “г" и “д"), или бо́льшего (диаграмма "е") числа фотонов. Вклад такого типа диаграмм в сечение рассеяния нельзя экспериментально отличить от вклада диаграмм "а"-"в", если суммарная энергия испущенных в тормозном излучении фотонов ниже порога чувствительности измерительного прибора. 
Расчеты показывают, что если учесть наряду с диаграммами "а"-“в" диаграммы "г" и "д", то инфракрасные расходимости компенсируются. Правда, при этом возникает зависимость сечения рассеяния от параметра, характеризующего чувствительность измерительного прибора. В качестве такого параметра $E_{\max }$ можно взять максимальную суммарную энергию дополнительно испущенных фотонов. Это вполне физический параметр, поэтому зависимость от него измеренного сечения рассеяния не может вызвать принципиального возражения. Однако учет только диаграмм "а"-“д” приводит к еще одной неприятности. Зависимость сечения рассеяния от параметра $E_{\max }$ оказывается сингулярной, и при достаточно малом значении $E_{\max }$ сечение становится отрицательным.

В настоящее время считается, что эта трудность является артефактом, связанным с использованием аппарата теории возмущений. Действительно, если рассмотреть следующие порядки теории возмущений, учесть вклад диаграмм типа "e" с нарастающим числом испущенных фотонов и просуммировать все эти вклады, то зависимость сечения от параметра $E_{\max }$ становится регулярной. Более того, при $E_{\max } \rightarrow 0$ сечение также стремится к нулю. С физической точки зрения это не вызывает возражения. Чисто упругое рассеяние, когда не испускается ни одного тормозного фотона, является одним из бесконечного числа каналов, по которому может пойти рассматриваемый процесс. Не удивительно, что каждый из этих каналов дает бесконечно малый вклад в суммарное сечение.

Здесь надо взглянуть по-новому на процесс, который мы называем упругим рассеянием. В действительности этот процесс не является чисто упругим. Рассеяние электрона всегда сопровождается тормозным излучением, которое не может быть зарегистрировано измерительным прибором даже с хорошей чувствительностью. Наряду с этим при чрезмерно хорошей чувствительности результат эксперимента станет сильно зависеть от этой чувствительности. При бесконечном увеличении чувствительности регистрируемое сечение упругого рассеяния должно стремиться к нулю. Поэтому в этом случае мы фактически будем исследовать не столько изучаемый физический объект (электрон), сколько измерительный прибор.

Из приведенного примера можно извлечь несколько полезных уроков.

УРок 1. Выделение характеристик изучаемого физического объекта несколько условно. Эти характеристики не могут быть совершенно отделены от характеристик измерительного прибора, с которым этот объект взаимодействует.

УРОк 2. Физический объект (в приведенном примере - электрон), который мы рассматриваем в качестве носителя определенных физических характеристик (дифференциального сечения упругого рассеяния), сопровождается полем (тормозными фотонами), которое само измерительным прибором не регистрируется, но влияет на результат измерения изучаемых характеристик.

УРок 3. Наличие сопровождающего поля не противоречит аксиомам локальности в квантовой теории поля. В приведенном примере электрон и тормозные фотоны распространяются в будущем световом конусе с вершиной в точке рассеяния.

6 Теоретическая и математическая физика, т. 155, № 2, 2008 г. 
УРок 4. Результат измерения характеристик (сечения рассеяния), приписываемых достаточно хорошо локализованному изучаемому объекту (электрону), может зависеть от характеристик физических объектов (тормозных фотонов), которые в момент измерения находятся в области, пространственноподобной относительно области локализации изучаемого объекта. Это может восприниматься как нелокальность изучаемого объекта.

Из приведенных уроков можно сделать следующий вывод. С точки зрения процесса измерения исследуемую физическую систему следует разделить на две части. Первая из них, которую в дальнейшем мы будем называть керном, регистрируется измерительным прибором. Вторая, которую мы будем называть темным полем, непосредственно измерительным прибором не регистрируется, однако от характеристик темного поля показание измерительного прибора зависеть может. Разделение на эти две части не является абсолютным, а зависит от используемой измерительной процедуры. В математическом аппарате квантовой теории поля эта подвижность границы отчасти находит свое отражение в формализме ренормгруппы.

В рамках описываемого в настоящей статье алгебраического подхода к квантовой теории такое разделение физической системы на две части можно связать с двумя элементами математического аппарата: алгеброй динамических величин и элементарным состоянием. Каждая квантовая частица проявляет себя через соответствующие наблюдаемые величины, точнее, через локальные наблюдаемые, значения которых могут быть найдены с помощью измерений, производимых в ограниченной области $\mathcal{O}$ пространства Минковского. Поэтому можно считать, что математическим образом квантовой частицы является локальная алгебра $\mathfrak{A}(\mathcal{O})$. В стандартном алгебраическом подходе к квантовой теории поля эту алгебру обычно называют алгеброй локальных наблюдаемых величин. Это не совсем правильно, так как множество наблюдаемых алгебры не образует, а лишь является подмножеством локальной алгебры соответствующих динамических величин.

Естественно считать, что область $\mathcal{O}$ является областью локализации рассматриваемой квантовой частицы. Во всяком случае, $\mathcal{O}$ должна содержать область локализации частицы. Если быть более точным, область локализации частицы следует ассоциировать с ее керном. Дело в том, что, как объяснялось выше, регистрируемые значения наблюдаемых могут зависеть от характеристик темного поля. Это поле не обязано быть локализованным в области $\mathcal{O}$. С другой стороны, в описываемом в настоящей статье подходе значение наблюдаемых определяется элементарным состоянием. Поэтому материальным носителем элементарного состояния следует считать не только керн рассматриваемого квантового объекта, но и ассоциированное с ним темное поле. Таким образом, элементарное состояние нельзя считать локализованным в области $\mathcal{O}$. Однако в отличие от квантового состояния, которое вообще не имеет никакой локализации в пространстве Минковского, элементарное состояние локализацию имеет: это локализация темного поля.

Отметим, что не всякое темное поле влияет на результат измерения наблюдаемых (в рассмотренном примере - на сечение рассеяния), а только то, которое родилось вместе с керном. То есть существенным является только темное поле, согласованное (когерентное) с керном. 
Вернемся теперь к обсуждению эксперимента по рассеянию на двух щелях. Будем считать, что рассеивается электрон. Мы получили формулу (12), которая описывает явление интерференции, используя тот факт, что квантовый ансамбль $\Psi_{a+b}$ имеет определенную структуру. Во-первых, в момент создания ансамбля электрон локализован либо в области щели $a$, либо в области щели $b$ (точнее, следовало бы говорить не об электроне, а о керне электрона). Во-вторых, в результате взаимодействия электрона со щелями $a$ и $b$ формируется ансамбль, структура которого такова, что соответствующий функционал $\Psi_{a+b}(\cdot)$ линеен.

Фактически мы заменили реальное описание взаимодействия электрона со щелями подходящими граничными условиями. Для математического описания рассматриваемого явления этих граничных условий достаточно. Однако хотелось бы, хотя бы на качественном уровне, выяснить, какие физические процессы стоят за этими граничными условиями.

Первое условие, локализация электрона, представляется самоочевидным и особого комментария не требует. Отметим только, что оно наверняка вызовет яростное возражение ортодоксальных сторонников стандартной квантовой механики, которые будут утверждать, что до измерения нельзя говорить о какой-либо локализации электрона. А почему нельзя? Только потому, что они не могут сказать что-нибудь вразумительное по этому поводу?

Для качественного объяснения второго условия можно предложить следующую модель взаимодействия электрона со щелями, точнее, с экраном, в котором эти щели прорезаны. При рассеянии к щелям подлетает не только керн электрона, но и сопровождающее его когерентное с ним темное поле. Поскольку это поле безмассовое, то оно подлетит даже раньше керна. Это поле возбудит в экране коллективные колебания, которые также будут когерентны керну. Возникшие колебания очень слабые, но в силу когерентности они могут резонансно взаимодействовать с керном. Во всяком случае, они могут сыграть роль случайной силы, которая примет участие в формировании вероятностного распределения импульса рассеяного электрона. В отличие от керна электрона, темное поле достигает обеих щелей, поэтому характер случайной силы существенно зависит от того, открыта одна из щелей или одновременно обе. Этот факт может явиться физической основой возникновения интерференционной картины.

В эксперименте темное поле проявляет себя при измерении значений наблюдаемых, которые описывают керн, когерентный этому полю. Оторвавшись от своего керна, темное поле становится непосредственно экспериментально не уловимым. Поэтому оно является хорошим кандидатом на роль конституента темной материи. Конечно, помимо электромагнитного поля, вклад в темное поле могут давать и другие безмассовые поля, такие как, например, глюонное и гравитационное поля.

Как уже говорилось ранее, чтобы непротиворечивым образом избавиться от инфракрасных расходимостей, нужно считать, что, например, при электромагнитных взаимодействиях излучается бесконечное число фотонов с ограниченной суммарной энергией. Такая система будет вести себя уже как классическое электромагнитное поле, а значит, безмассовые наблюдаемые квантовые поля должны иметь классические "хвосты". Это открывает интересную перспективу для гравитационного поля. 


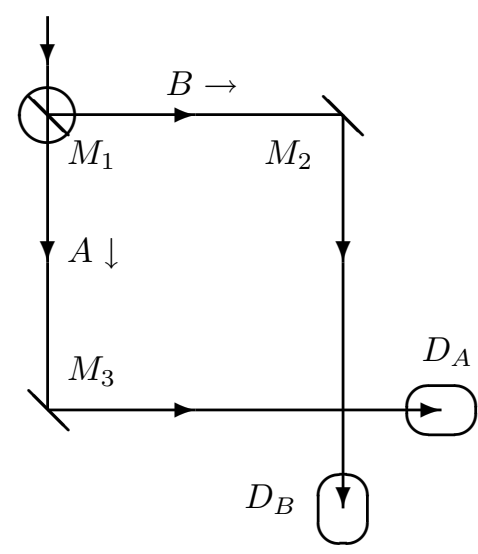

$a$

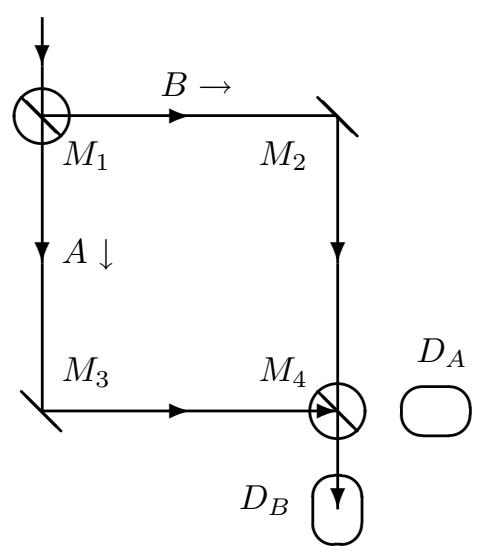

$\sigma$

Рис. 2. Эксперимент с отсроченным выбором.

Несмотря на многочисленные попытки, до сих пор не удалось построить непротиворечивую квантовую модель гравитационного поля. Кванты гравитационного поля, гравитоны, никто не наблюдал. Может быть гравитационное поле состоит только из классического "хвоста"?

Механизм темного поля позволяет очень наглядно объяснить результат эксперимента с так называемым отсроченным выбором. Идею этого эксперимента тридцать лет назад предложил Вилер [17]. Почти идеальную реализацию предложения Вилера удалось осуществить совсем недавно [18]. Реальный эксперимент полностью подтвердил предсказания Вилера.

Принципиальная схема экспериментальной установки изображена на рис. 2. На нем $M_{2}$ и $M_{3}$ - два полностью отражающих зеркала, $M_{1}$ и $M_{4}$ - два полупрозрачных зеркала. Зеркало $M_{4}$ съемное. По желанию экспериментатора оно может отсутствовать (установка находится в положении “а") или присутствовать (установка находится в положении "б"). На зеркало $M_{1}$ подаются одиночные фотоны с временны́ми интервалами такими, что одновременно в установке может находиться не более одного фотона. После прохождения установки фотон попадает либо в детектор $D_{A}$, либо в детектор $D_{B}$.

Если установка находится в положении "а", и фотон ведет себя как частица, то после прохождении зеркала $M_{1}$ он выбирает с равными вероятностями либо маршрут $A$, либо маршрут $B$. В результате после прохождения установки он попадает либо в детектор $D_{A}$, либо в детектор $D_{B}$.

Если установка находится в положении "б", и фотон ведет себя как волна, то процесс прохождения фотона через установку можно представить следующим образом. 
Фотон-волна падает на зеркало $M_{1}$. Здесь волна расщепляется на две когерентные части. Одна идет по маршруту $A$, другая - по маршруту $B$. При этом когерентность частей сохраняется. Фаза волны при отражении от каждого зеркала изменяется на $\pi / 2$, при прохождении сквозь зеркало фаза не изменяется. В зеркале $M_{4}$ происходит когерентное сложение обеих частей волны. Баланс изменения фаз таков, что после зеркала $M_{4}$ волна будет распространяться только в направлении детектора $D_{B}$.

Результат реального эксперимента таков. Если установка находится в положении "а", то с вероятностью 0.5 срабатывает детектор $D_{A}$, и с такой же вероятностью детектор $D_{B}$. Если установка находится в положении “б", то с вероятностью 1 срабатывает детектор $D_{B}$. Таким образом, в зависимости от положения установки фотон ведет себя либо как частица, либо как волна. Такое поведение фотона находится в согласии с концепцией контекстуальности, предложенной Бором [5]. Согласно этой концепции результат квантового эксперимента зависит от общего контекста эксперимента.

Однако Вилер предложил усложнить для фотона проблему выбора. Он предложил вставлять или убирать зеркало $M_{4}$ после того, как фотон уже прошел зеркало $M_{1}$, т.е. фотон должен предугадать последующие действия экспериментатора. Реальный эксперимент показал, что фотон успешно справляется с этой задачей и в каждой ситуации ведет себя нужным образом: либо как частица, либо как волна.

В этом эксперименте вроде бы проявляется нелокальность во времени: будущее действие (манипуляции с зеркалом $M_{4}$ ) влияет на предыдущее действие (выбор поведения фотона как волны или частицы). Сам Вилер интерпретировал результат эксперимента как подтверждение концепции "нет регистрации результата эксперимента - нет физического явления".

В рамках механизма темного поля объяснение результата эксперимента гораздо более простое. При взаимодействии падающего фотона с зеркалом $M_{1}$ помимо рассеяния (отражения или прохождения) фотона рождаются дополнительные тормозные фотоны. Керн рассеянного фотона в зависимости от элементарного состояния последнего распространяется либо по маршруту $A$, либо по маршруту $B$. Тормозные фотоны (темное поле) распространяются по обоим маршрутам. Обе части темного поля достигают зеркала $M_{4}$ (если оно присутствует), где когерентно складываются и возбуждают малые коллективные колебания в зеркале $M_{4}$. Эти малые колебания когерентны керну фотона, они взаимодействуют с ним резонансным образом и выступают в роли случайной силы, которая направляет керн в сторону детектора $D_{B}$. Если зеркало $M_{4}$ отсутствует, то керн фотона движется по одному из маршрутов, который он выбрал в зеркале $M_{1}$, и попадает либо в детектор $D_{A}$, либо в детектор $D_{B}$. Никакой нелокальности во времени здесь нет.

K проблеме локальности тесно примыкает проблема квантовых корреляций. Дело в том, что во многих случаях эти корреляции выглядят как результат действия на расстоянии. Типичным примером здесь является парадокс Эйнштейна-Подольского-Розена [1]. Например, в варианте, предложенном Бомом [19], результат измерения проекции спина на некоторое направление у одной частицы из синглетной пары частиц со спинами $1 / 2$ мгновенно и однозначно предсказывает результат измерения проекции спина на то же направление у второй частицы, удаленной на значительное 
расстояние. Кажется, что такой результат резко противоречит принципу локальности. Однако это противоречие имеет место только в том случае, если считать, что корреляция возникает за счет взаимодействия между этими частицами в момент измерения.

Представление о том, что корреляции между отдельными элементами физической системы всегда обусловлены каким-то взаимодействием между этими элементами, является прочно укоренившимся заблуждением. Оно даже получило свое отражение в терминологии, используемой в квантовой механике. Например, часто можно услышать слова "обменное взаимодействие", "несиловое квантовое воздействие" или рассуждения о "сильных квантовых корреляциях".

В действительности квантовые корреляции вовсе не обусловлены какими-то специфическими свойствами квантовых взаимодействий. Например, в парадоксе Эйнштейна-Подольского-Розена причиной корреляции между проекциями спинов двух частиц является то, что они родились как синглетная пара, для которой справедлив закон сохранения собственного момента импульса. Закон сохранения момента импульса справедлив и в классической физике.

В большинстве случаев квантовые корреляции обусловлены структурой ансамбля физических систем, с которым приходится иметь дело в квантовых экспериментах. Эта структура фиксируется процедурой приготовления рассматриваемого ансамбля, а процедура приготовления определяется свойствами используемого классического прибора. Таким образом, для квантовых корреляций, как правило, важны не взаимодействия между квантовыми объектами, а взаимодействие каждого отдельного члена квантового ансамбля с приготавливающим квантовый ансамбль классическим прибором (или приборами). Это взаимодействие для отдельных членов ансамбля может быть разнесено как во времени, так и пространстве. Поэтому нет ничего удивительного в том, что очень часто кажется, что корреляции противоречат свойству локальности взаимодействия. В действительности свойство локальности для корреляций всегда выполняется. Только эту локальность надо проверять не с точки зрения взаимодействия между членами квантового ансамбля. Ее надо оценивать с точки зрения взаимодействия отдельных членов этого ансамбля с приготавливающими ансамбль приборами.

\section{4. ЗАКЛЮЧЕНИЕ}

Подводя итоги, можно сделать следующие выводы.

Квантовую теорию, и релятивистскую, и нерелятивистскую, можно сформулировать таким образом, что она не будет противоречить условию локальности, принятому в квантовой теории поля. Процесс измерения также не противоречит этому условию.

Отмеченная Эйнштейном неполнота квантовой механики устраняется с помощью введения нового понятия “элементарное состояние”, которое является атрибутом индивидуальной физической системы.

С точки зрения процедуры измерения исследуемая физическая система может быть разложена на две части: так называемые керн и сопровождающее темное поле. Керн является материальным носителем корпускулярных свойств физической 
системы. Керн локализован в пространстве Минковского. Роль математического образа керна выполняет алгебра локальных наблюдаемых. Структура темного поля не противоречит релятивистскому условию локальности, но темное поле имеет худшую локализацию, чем керн. Элементарное состояние физической системы определяется как структурой керна, так и структурой темного поля. Элементарное состояние является математическим образом материального носителя волновых свойств физической системы.

Квантовое состояние является классом эквивалентности в множестве элементарных состояний и играет роль математического образа (квантового) ансамбля исследуемых физических систем. Квантовое состояние не обладает свойством локальности в пространстве Минковского.

\section{Список литературы}

[1] А. Эйнштейн, Б. Подольский, Н. Розен, УФН, 16:4 (1936), 440-446.

[2] А. Эйнштейн, "Физика и реальность”, Физика и реальность, Сб. ст., перевод. Сост. и коммент. У.И. Франкфурт, Наука, М., 1965, 38.

[3] А. Эйнштейн, “Основы теоретической физики”, Физика и реалъность, Сб. ст., перевод. Сост. и коммент. У. И. Франкфурт, Наука, М., 1965, 67.

[4] Н. Бор, УФН, 16:4 (1936), 446-457.

[5] Н. Бор, "Дискуссия с Эйнштейном по проблемам теории познания в атомной физике", Избр. науч. труды, Т. 2, статьи (1925-1961), ред. И. Е. Тамм, В. А. Фок, Б. Г. Кузнецов, Наука, М., 1971, 399-433.

[6] Р. Стритер, А.С. Вайтман, РCT, спин и статистика и все такое, Наука, М., 1966.

[7] Н. Н. Боголюбов, Д. В. Ширков, Введение в теорию квантованных полей, Наука, М., 1984.

[8] R. Haag, D. Kastler, J. Math. Phys., 5:7 (1964), 848-861.

[9] H. Araki, Progr. Theoret. Phys., 32:5 (1964), 844-854.

[10] И. Фон Нейман, Математические основы квантовой механики, Наука, М., 1964.

[11] Д. А. Славнов, ТМФ, 149:3 (2006), 457-472.

[12] Д. А. Славнов, ЭЧАЯ, 38:2 (2007), 295-359.

[13] А.Н. Колмогоров, Основные понятия теории вероятностей, Наука, М., 1974.

[14] Ж. Неве, Математические основы теории вероятностей, Мир, М., 1969.

[15] Ж. Диксмье, $C^{*}$-алгебры и их представления, Наука, М., 1974.

[16] М. Пескин, Д. Шредер, Введение в квантовую теорию поля, РХД, Ижевск, 2001.

[17] J. A. Wheeler, "The "past" and the "delayed-choice" double-slit experiment", Mathematical Foundations of Quantum Theory, Presentations made to a Conference at Loyola University (New Orleans, La., June 2-4, 1977), ed. A. R. Marlow, Academic Press, New York, 1978, $9-48$.

[18] V. Jacques, E. Wu, F. Grosshans, F. Treussart et al., Experimental realization of Wheeler's delayed-choice GedankenExperiment, arXiv: quant-ph/0610241.

[19] Д. Бом, Квантовая теория, Наука, М., 1965.

Поступила в редакцию 6.03.2007 